\title{
Direct effects of dopamine on mitochondrial thermogenesis in brown adipocytes
}

\section{Rose Kohlie, Nina Perwitz, Hendrik Lehnert, Johannes Klein, Alexander Iwen}

Department of Internal Medicine I, University of Lübeck, Germany

Background: Brown adipose tissue (BAT) is specialized in thermogenesis, i.e. the production of heat. Uncoupling protein 1 (UCP1) mediates this effect in mitochondria of brown adipocytes [1]. Catecholamines are known to be critically involved in the regulation of BAT thermogenesis [2]. However, little is known about dopamine (DA)mediated effects on thermogenesis. Here, we investigated direct cellular effects of DA on mitochondrial thermogenesis and mass in brown adipocytes.

Methods: Cell culture: SV-40T immortalized murine brown adipocytes were used for all experiments.

Western blot: Protein lysates were prepared and immunoblotting was performed using specific antibodies. Mature brown adipocytes were treated with dopamine (DA; 1 or $10 \mathrm{nM})$ for $24 \mathrm{~h}$ in serum free medium. cAMP determination: Brown adipocytes were treated as stated in the figure. Cells were lysed and supernatants were analyzed using an ELISA kit (Caymen Chemical, Hamburg, Germany)

Oxygen consumption: Respiration was measured using Oxoplates (FluoSTAR OPTIMA; BMG-Labtech, Ortenberg, Germany).

JC-10 assay: Mitochondrial membrane potential $\left(\Delta \psi_{\mathrm{m}}\right)$ was analyzed with the JC-10 dye (Biomol, Hamburg, Germany). Fluorescence intensities of $\mathrm{JC}-10$ monomers and aggregates were quantified using a microplate reader

Statistical analysis: Paired Student's $t$-test was performed using Sigma Plot ${ }^{* *} p<0,01 ;{ }^{*} p<0,05$

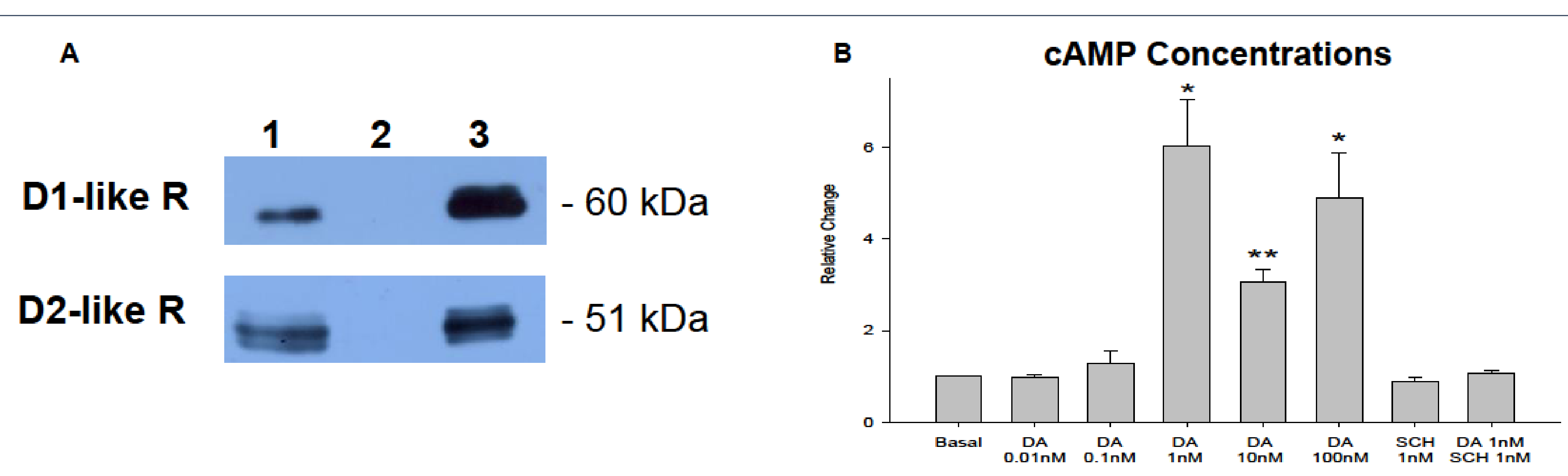

Fig.1: Western blot for D1-like and D2-like dopamine receptors expression (A) (1= BAT cell line, $2=$ negative control, $3=$ positive control). DA treatment of brown adipocytes for 2 minutes causes a dosedependent increase of cAMP concentrations. D1-like receptor antagonist SCH $23390(\mathrm{SCH})$ abolished the effect of DA on cAMP level, SCH alone had no effect on cAMP levels (B).
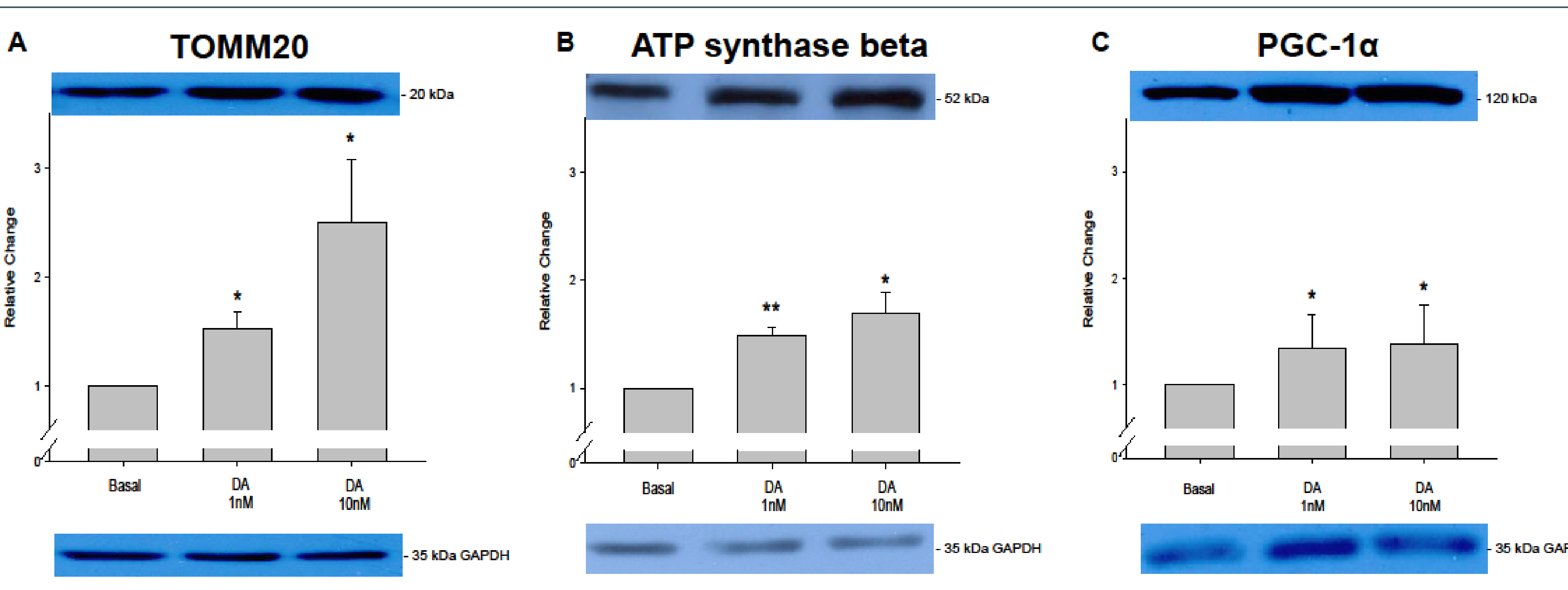

Fig.3: Dopamine (DA) increases mitochondrial mass: DA treatment of brown adipocytes for 24 hours increases expression of two mitochondrial mass marker TOMM20 (A) and ATP synthase beta (B) dose dependently also DA induces expression of PGC-1a (C) in BAT and ATP synthase beta $(B)$ dose dependently also DA induces
significantly to basal (PGC-1 a = peroxisome proliferator-activated receptor y coactivator $1 a)$
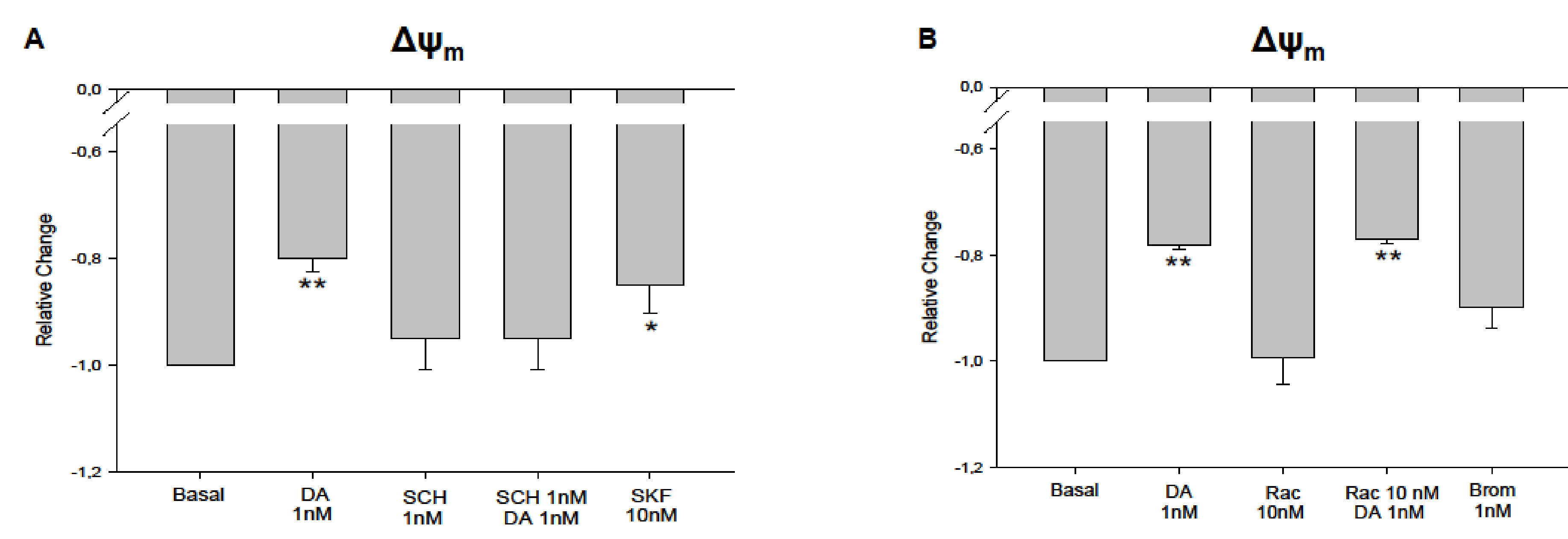

Fig.4: Dopamine (DA) effects on $\Delta \Psi_{m}$ are mediated by dopamine 1-like receptors: Treatment of brown adipocytes with DA significantly increases $\Delta \psi_{\mathrm{m}}$ within 24 hours. A D1-like receptor antagonist ( $\mathrm{SCH}$ ), but not the D2-like receptor antagonist Raclopride (Rac), abolished the DA-effect on $\Delta \psi_{\mathrm{m}}$. The specific D1-like receptor agonist SKF 38393 (SKF) had a similar effect on $\Delta \psi_{\mathrm{m}}$ as DA, whereas bromocriptine (Brom), a D2-like receptor agonist, had no significant effect $(A, B)$.
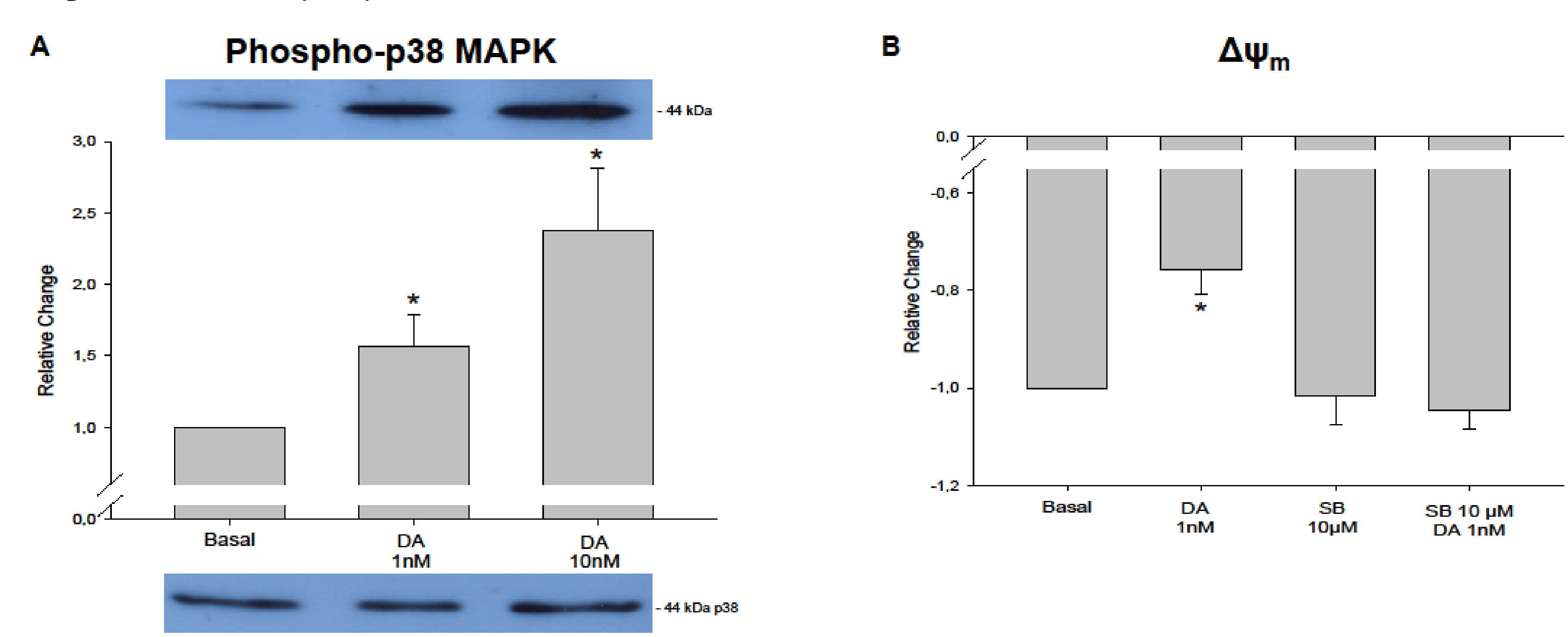

Fig.5: Dopamine (DA) effects on $\Delta \Psi_{m}$ are p38 MAPK-dependent: Treatment of brown adipocytes with DA significantly increases p38 MAPK phosphorylation (A) and pharmacological inhibition of p38 MAPK with specific inhibitor SB 202190 (SB) abolished the DAmediated effect on $\Delta \psi_{m}(B)$.
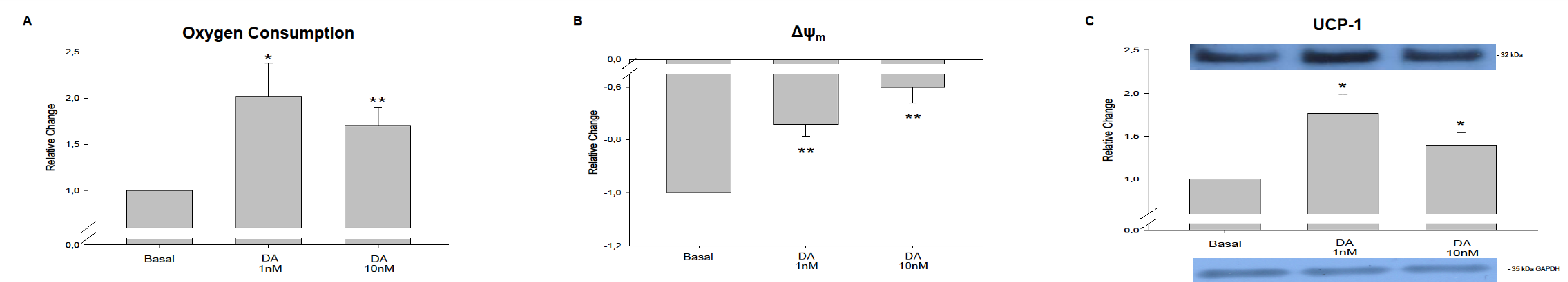

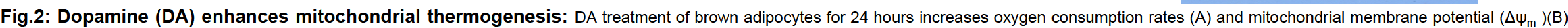
Protein levels of UCP-1 increase significantly upon treatment with DA (C).

\section{Summary:}

D1- like and D2- like dopamine receptors were detectable in brown adipocytes. Treatment of brown adipocytes with dopamine:

\section{- increased cAMP concentrations \\ increased oxygen consumption, $\Delta \psi_{\mathrm{m}}$ and UCP-1 levels \\ increased expression of mitochondrial mass markers}

These direct effects of dopamine on mitochondrial thermogenesis were mediated by D1-like receptors and p-38 MAP kinase.

\section{Conclusion:}

Dopamine directly increased thermogenesis in brown adipocytes Targeting D1-like receptors on brown adipocytes may help to induce thermogenesis, pointing towards novel therapeutic approaches to treat obesity.

References:

[1] Kozak LP, Koza RA, Anunciado-Koza R (2010) Int J Obes. 34 Suppl 1:S23-7. [2] Collins S, Yehuda-Shnaidman E, Wang H (2010) Int J Obes. 34 Suppl 1:S28-33. 